\title{
Guest editors' foreword to the special issue on Virtual Reality in Scientific Application
}

\author{
Osvaldo Gervasi · Roberto Ranon
}

Received: 14 October 2009/ Accepted: 20 October 2009/Published online: 5 November 2009

(C) Springer-Verlag London Limited 2009

In the last decades, virtual environments are (perhaps slowly, but steadily) moving from Virtual Reality research laboratories to several application domains, where they have become useful tools for study, exploration, and discovery. This special issue of Virtual Reality is devoted to illustrate various applications that employ Virtual Reality for scientific purposes. The special issue comprises ten papers, thanks goes to the careful reviewing effort by the members of a multidisciplinary scientific committee, which included experts in the fields of Virtual Reality, as well as in the different scientific domains. For reasons of space, this special issue is split into two volumes.

The present volume consists of five papers, which are briefly presented in the followings.

The paper "Visual Immersive Haptic Mathematics" by Alexei Sourin and Lei Wei presents an approach that allows one to define complex geometry, visual appearance, and tangible physical properties of virtual objects using the language of mathematical functions and proposes to use this as a tool in mathematical education. Their proposal is based on extending the well-known VRML and X3D standards with function-based nodes.

O. Gervasi $(\bowtie)$

Department of Mathematics and Computer Science,

University of Perugia, Via Vanvitelli, 1, 06123 Perugia, Italy

e-mail: osvaldo@unipg.it

\section{R. Ranon}

Human-Computer Interaction Lab, Department of Mathematics and Computer Science, University of Udine, Via delle Scienze 206, 33100 Udine, Italy

e-mail: ranon@dimi.uniud.it
The paper "Tangible Props for Scientific Visualization: Concept, Requirements, Application,” by Krzysztof Jakub Kruszyński and Robert van Liere explores the use of printed tangible props (produced using three-dimensional printing technology) as input devices for scientific visualization.

The paper "Immersive Structural Biology: A New Approach to Hybrid Modeling of Macromolecular Assemblies" by Jochen Heyd and Stefan Birmanns describes a virtual environment for true interaction with and solution of hybrid biomolecular modeling problems, allowing the user to efficiently solve even difficult multiresolution docking problems.

The papers "Multisensory VR exploration for Computer Fluid Dynamics in the CoRSAIRe project" by J. M. Vezien et al. and the paper "Multisensory VR interaction for Protein-Docking in the CoRSAIRe project" by N. Ferey et al. describe the usage of Virtual Reality to perform the analysis of large and complex datasets. The first paper describes the multimodal VR environment implemented to immersively study the Computational Fluido Dynamics data. The second paper describes the VR environment implemented to study the protein docking where visual, audio, and haptic feedbacks are combined to render and evaluate chemical or physical properties of a given docking configuration.

We would like to mention all the reviewers of this special issue: Tomaz Amon, Suneth Attygalle, Marc Baaden, Fernando Bello, Katia Buehler, Stefano Burigat, Fabio Buttussi, Marinella Cadoni, Luca Chittaro, Demis Corvaglia, Judith Deutsch, Cali Fidopiastis, Christian Florian, Ivan Gerace, Anthony Jameson, Nigel John, Dieter Kranzlmueller, Riccardo Magni, Kresimir Matkovic, Pascal Mimero, Torsten Moeller, Taha Mzoughi, Dimitri Plemenos, Genaro Rebolledo-Mendez, Maryiam 
Shamsuddin Siti, Erik Van der Spek, Milos Sramek, Mario Valle, Alberto Cabas Vidani, Chad Wingrave, Kin Hong Wong, and Mauro Zampolini. We thank them all for spending their time, energy, and competence to shape this special issue.
We hope that readers will find this special issue a source of valuable information regarding the current state of the art in scientific applications of Virtual Reality.

Perugia and Udine, September 20, 2009. 